\title{
Treatment with an inhibitor of catechol-O-methyltransferase activity reduces preterm birth and impedes cervical resistance to stretch in pregnant rats
}

\author{
Melissa J Wentz, Shao-Qing Shi, Leili Shi, Salama A Salama, Hassan M Harirah, Hala Fouad, \\ Robert E Garfield and Ayman Al-Hendy
}

Department of Obstetrics and Gynecology, University of Texas Medical Branch, 301 University Boulevard, Galveston, Texas 77555-0587, USA

Correspondence should be addressed to A Al-Hendy; Email: ahendy@mmc.edu

\begin{abstract}
Catechol-O-methyltransferase (COMT) enzyme catalyzes the methylation of the 2- or 4-hydroxyestrogens to 2- or 4-methoxyestrogens. Both the hydroxyestrogens and methoxyestrogens have been shown to block or enhance the effects of estrogen respectively. Our objective was to investigate the potential role of COMT in parturition and cervical ripening using a rat model. Immunohistochemistry was conducted to detect and localize the COMT protein in rat uterine tissues during pregnancy. We measured the longitudinal changes in urinary 2-hydroxyestrogen before, during, and after pregnancy in rats. Animal studies were conducted to determine the effect of treatment with a selective COMT inhibitor on (1) mifepristone-induced preterm birth and (2) cervical resistance to stretch in pregnant rats. The intensity of staining for the COMT protein differed within the luminal epithelium, uterine gland epithelium, endometrium, and myometrium during pregnancy. Levels of staining for the COMT protein in rat myometrium were highest on day 1 and lowest on days 8 and 13, but high levels returned by days 16 and 19 of pregnancy. The levels of urinary 2-hydroxyestrogen gradually increased in the first 2 weeks of pregnancy, peaked from days 16 to 18 of pregnancy, and then gradually returned to pre-pregnancy levels after delivery. The percentage of pups retained in the uterus of pregnant rats treated with both mifepristone and COMT inhibitor $(\mathbf{4 8} \pm \mathbf{1 5} \%)$ was significantly higher $(P<0.05)$ when compared with the value of pregnant rats treated with mifepristone alone $(12 \pm 4 \%)$. The resistance to stretch was significantly higher $(\boldsymbol{P}<\mathbf{0 . 0 5})$ in cervical tissues from the pregnant rats treated with COMT inhibitor $(0.28)$ when compared with cervical tissues taken from rats treated with vehicle control (0.18). Modulation of COMT activity may play a role in the regulation of myometrial contractility and cervical ripening during pregnancy.

Reproduction (2007) 134 831-839
\end{abstract}

\section{Introduction}

Estrogen and progesterone play key roles in maintaining pregnancy and initiating parturition by modifying myometrial contractility and excitability (Csapo \& Pinto-Dantas 1965, Challis 2000). It is well accepted that progesterone mediates myometrial quiescence (Csapo \& Pinto-Dantas 1965) while estrogen stimulates changes that increase myometrial contractility (Nathanielsz 1995, Challis 2000). Near term, the myometrium transforms from a quiescent to a contractile state. In many mammalian species, this transformation is achieved by a decrease in circulating progesterone and a concurrent rise in estrogen levels (Casey \& MacDonald 1988, Liggins 1994). In humans, however, the mechanisms for progesterone withdrawal and estrogen activation are not understood because the circulating levels of progesterone and estrogen remain high during pregnancy and throughout parturition.
Progesterone supports uterine quiescence during pregnancy by several mechanisms. Progesterone suppresses the expression of proteins that help to mediate uterine contractility such as connexin (CX) 43 (Garfield et al. 1988), calcium channels (Tezuka et al. 1995), and receptors for uterotonins (Dong \& Yallampalli 2000). Progesterone also supports uterine quiescence by up-regulation of pathways associated with relaxation like the nitric oxide system (Garfield et al. 1998), which promotes synthesis of intracellular cyclic guanosine 3',5'-monophosphate (GMP; Buhimschi et al. 1995). Finally, progesterone also inhibits production of uterotonins like prostaglandin F2 $\alpha$ (PGF2 $\alpha$; Garfield et al. 1998, Farina et al. 2004).

In contrast to progesterone, estrogen plays an important role in preparing the myometrium for parturition (Nathanielsz 1995, Challis 2000). At term pregnancy, estrogen helps alter the biochemical and electrical properties of the myometrium by increasing 
levels of the proteins that are directly or indirectly involved in muscle contraction such as CX 43 (Mackenzie \& Garfield 1986, Oltra et al. 2003), and the $\alpha 1$ and $\beta$ ( $\beta 1)$ subunits of the L-type voltagedependent calcium channel (Tezuka et al. 1995). In addition, estrogen may facilitate myometrial contractility by increasing the expression levels of oxytocin receptor (OTR; Fuchs et al. 1983, Larcher et al. 1995, Fang et al. 1996) and the FP receptor that binds PGF2 $\alpha$ (Dong \& Yallampalli 2000).

Catechol-O-methyltransferase (COMT) catalyzes the transfer of a methyl group from $S$-adenosyl-methionine to one of the phenolic hydroxyl groups of a variety of catechols, including catecholestrogens and catecholamine neurotransmitters (Axelrod \& Tomchick 1958, Creveling 2003). In estrogen metabolism, COMT converts the catecholestrogens, 2 - and 4 hydroxyestrogen, to 2- and 4-methoxyestrogen respectively (Creveling 2003). The catecholestrogens, 2- or 4-hydroxyestrogen, can act as estrogen antagonists (Vandewalle \& Lefebvre 1989, Bradlow 1996, Al-Hendy \& Salama 2006) while their methylated counterparts, 2- or 4-methoxyestrogen, can act as estrogen agonists in multiple biologic assays (Banerjee et al. 2003, Lippert et al. 2003, Liu \& Zhu 2004, Sutherland et al. 2005). COMT is present in many types of tissues, including those involved in reproduction, such as the placenta (Castren \& Saarikoski 1974, Barnea et al. 1988), the decidua vera (Casey \& MacDonald 1983), the myometrium (Al-Hendy \& Salama 2006, Wentz et al. 2006), and the endometrium (EN; Briggs \& Briggs 1973, Casey \& MacDonald 1983, Al-Hendy \& Salama 2006, Salih et al. 2007a). High levels of COMT activity are present in placental and decidua vera samples obtained from pregnant women at term (Castren \& Saarikoski 1974, Casey \& MacDonald 1983, Barnea et al. 1988). COMT activity or expression levels in endometrial tissues are cycle-dependent (Casimiri et al. 2004, Salih et al. 2007a) and modulated by steroid hormones (Casimiri et al. 2004, Salih et al. 2007a).

The formation and metabolism of catecholestrogens may have multiple roles in reproduction. Treatment with catecholestrogens stimulates progesterone synthesis in porcine granulosa cells (Spicer \& Hammond 1988, 1989) and in luteal cells taken from day 8 pseudopregnant rats (Tekpetey \& Armstrong 1994). Treatment with catecholestrogens helps to mediate implantation in rodents (Hoversland et al. 1982, Kantor et al. 1985, Chakraborty et al. 1990), possibly by facilitating production of PGs (Pakrasi \& Dey 1983). It was proposed that the secretion of catecholestrogens by the conceptus may further establish pregnancy by preventing luteolysis (Geisert et al. 1990). Finally, in bovine uterine arteries, an interaction between catecholestrogens and potential sensitive channels is believed to cause an increase in blood flow to the uterus during pregnancy (Ford et al. 1992). Since COMT activity balances cellular levels of catecholestrogens, it may indirectly help to regulate these events.

Our objective in this study was to investigate the role of COMT in parturition and cervical ripening using a rat model. Our experimental approach included: (1) localization of COMT protein in uterine tissues taken from pregnant female rats at different days of pregnancy by immunohistochemistry; (2) longitudinal measurement of the urinary 2-hydroxyestrogen levels before, during, and after pregnancy in female rats; and (3) investigation of the effect of a selective COMT inhibitor on preterm birth (PTB) and cervical resistance to stretch in timed-pregnant rats.

\section{Results \\ Immunohistochemistry}

Results from the immunohistochemistry analysis suggested that COMT was differentially expressed in the rat uterus during pregnancy (Fig. 1). A similar pattern of staining for the COMT protein was present within the luminal epithelium (LE) and the epithelium layer of the uterine glands during pregnancy (Fig. 1), with intense staining for COMT protein at days 1, 2, and 19, and moderate staining at days $4,8,13$, and 16 of pregnancy (Fig. 1). This was in contrast to the weak staining for the COMT protein within the EN (Fig. 1). The pattern of staining for COMT protein in the myometrium was similar in both the circular and longitudinal smooth muscle cells (Fig. 1). The intensity of staining for COMT protein in the myometrium was highest on days 1 and 2 of pregnancy, decreased gradually, and was lowest on day 8 and 13 pregnant rats. Staining for COMT protein in the myometrium increased again by day 16 of pregnancy and remained high for the rest of pregnancy (Fig. 1). No staining was detected in the negative control samples in which the primary antibody was omitted during the immunohistochemistry procedure.

We used a scoring system to quantify the relative levels of COMT expression in the rat myometrium during pregnancy. They represent the intensity of staining with a score of 0 indicating no staining and 5 indicating intense staining. Average scores for each day of pregnancy analyzed in the study are listed in Table 1 . The average staining score for COMT protein in the myometrium was highest in uterine tissues obtained from day 1 $(3.57+0.271)$ and day $2(3.5+0.634)$ pregnant rats. These scores differed significantly from the average staining score for COMT in the myometrium from day $8(2.15+0.299)$ pregnant rats. The average staining scores for COMT protein were higher for late pregnant rats (day 19, 2.5 +0.265; day 22, 2.45+0.744) when compared with those for mid-pregnant rats (day 8, $2.15+0.299$; day $13,2.66+0.530)$. 

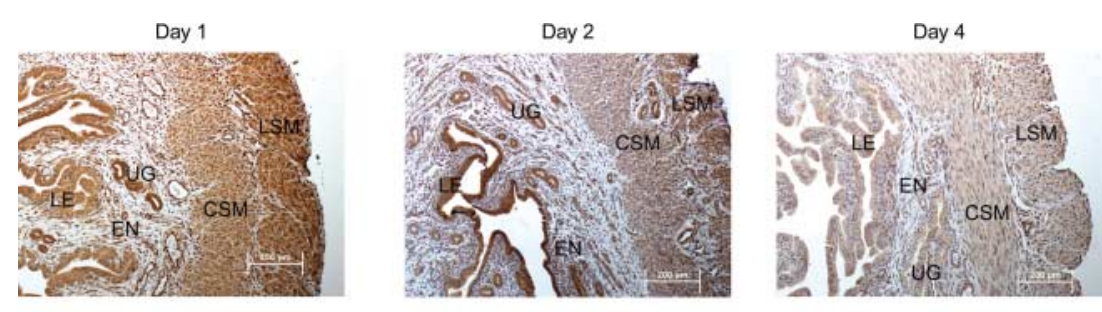

Day 8
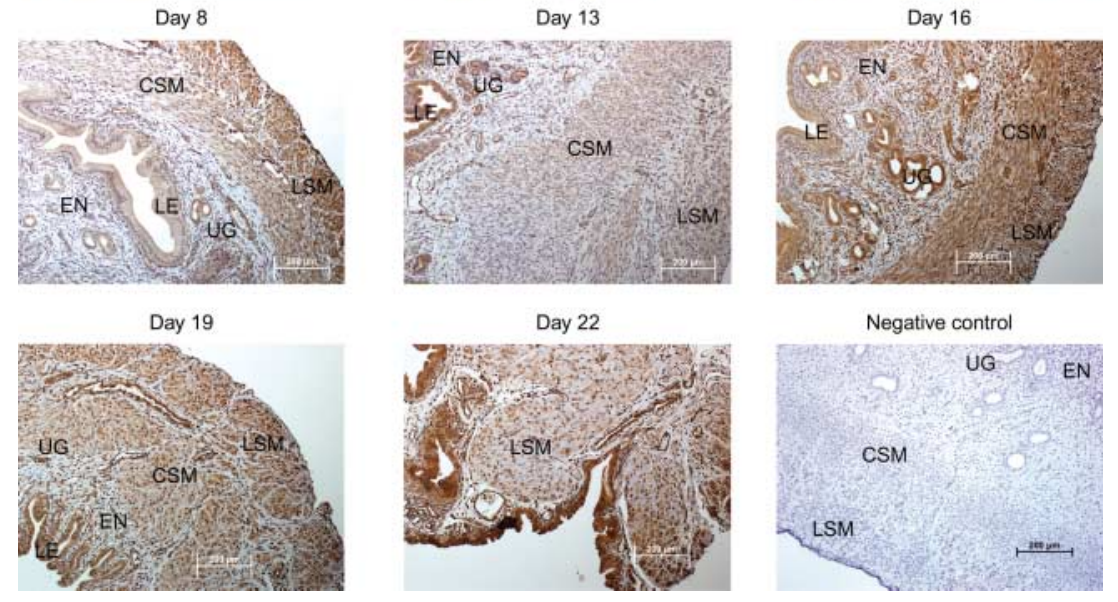

Figure 1 COMT expression in rat uterine tissue during pregnancy. Uterine tissues were collected from female Sprague-Dawley rats timed at days 1, 2, 4, 8, 13, 16, 19, and 22 (indicated in each picture) of pregnancy. Tissues were fixed, embedded in paraffin, and then sectioned. COMT protein was detected by immunohistochemistry using standard techniques. Negative control is shown in which anti-COMT antibody was omitted during the procedure using tissues collected from a nonpregnant rat. LE, luminal epithelium; UG, uterine glands; EN, endometrium; CSM, circular smooth muscle; LSM, longitudinal smooth muscle. Magnification: $100 \times$. Scale bars represent $200 \mu \mathrm{M}$.

\section{Longitudinal measurement of the urinary 2-hydroxyestrogen}

Figure 2 depicts the levels of urinary 2-hydroxyestrogen in nonpregnant, pregnant, and post partum rats. The levels of 2-hydroxyestrogen gradually increased in the first 2 weeks of pregnancy and subsequently peaked from day 16 to 18 of pregnancy. This was followed by a gradual decrease and return to pre-pregnancy levels after delivery (Fig. 2). The average levels of urinary 2-hydroxyestrogen detected in day $16(0.89 \pm 23), 17(0.95 \pm 21)$, and $18(0.96 \pm 16)$ pregnant rats were significantly higher $(P<0.05)$ when compared with average levels detected in nonpregnant rats $(0.30 \pm 0.07)$, pregnant rats (until day 10 of pregnancy, $0.47+0.06$ ), and post partum rats (day $4,0.4 \pm 0.05$; day 6 , $0.37 \pm 0.01$; Fig. 2).

\section{Effect of treatment with RO 41-0960 on PTB}

Figure 3 shows the average percentage of pups retained (PPR) in the uterus for pregnant rats in each treatment group. No PTBs were noted in pregnant rats treated with vehicle control or COMT inhibitor alone at either the $150 \mathrm{mg} / \mathrm{kg}$ or $75 \mathrm{mg} / \mathrm{km}$ dosages (Fig. 3). The PPR values for these treatment groups differed significantly from PPR values for groups treated with mifepristone alone or mifepristone and the COMT inhibitor at either the dose of 150 or $75 \mathrm{mg} / \mathrm{kg}$ (Fig. 3). The PPR value for pregnant rats treated with mifepristone alone was $12.2+3.6 \%$. Cotreatment of pregnant rats with mifepristone and COMT inhibitor at the $150 \mathrm{mg} / \mathrm{kg}$ dose caused an increase in the PPR value $(48.2+15.5 \%)$ and significantly differed from the value obtained from the pregnant rats treated with mifepristone alone $(P<0.001)$. However, cotreatment of pregnant rats with mifepristone and the COMT inhibitor at the $75 \mathrm{mg} / \mathrm{kg}$ dose did not cause an increase in the PPR $(12.36+5.8 \%)$. The litter size and body weight of the pups were not statistically different among all tested groups (data not shown).

\section{Effect of treatment with RO 41-0960 on cervical resistance to stretch during pregnancy}

Figure 4 shows the average value of cervical resistance to stretch in pregnant rats treated with either the COMT inhibitor $(0.284 \pm 0.021)$ or vehicle control $(0.183 \pm 0.027)$. The difference in values between the two groups was significant $(P=0.008)$. The values of cervical resistance to stretch in the vehicle control rats at day 19 of pregnancy was similar to values previously

Table 1 Average staining intensity scores for catechol-O-methyltransferase (COMT) protein in pregnant rat myometrium.

\begin{tabular}{lc}
\hline Day of pregnancy & Average score \pm s.E.M. \\
\hline Day 1 & $3.57 \pm 0.271$ \\
Day 2 & $3.5 \pm 0.634$ \\
Day 4 & $2.73 \pm 0.359$ \\
Day 8 & $2.15 \pm 0.299$ \\
Day 13 & $2.66 \pm 0.530$ \\
Day 16 & $3.03 \pm 0.612$ \\
Day 19 & $2.5 \pm 0.265$ \\
Day 22 & $2.45 \pm 0.744$ \\
\hline
\end{tabular}

S.E.M., standard error of the mean

aThe scores for day 1 and day 2 pregnant rats significantly differed from the score for day 8 pregnant rats. 


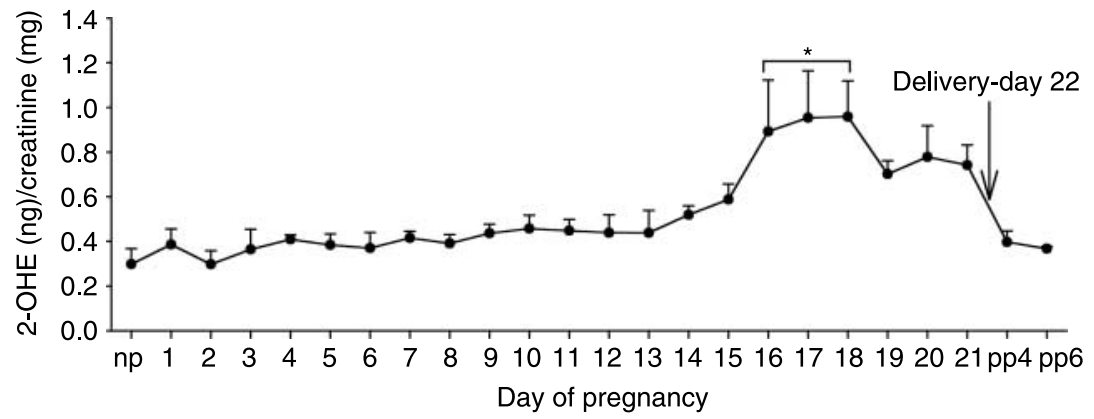

Figure 2 Longitudinal changes in urinary 2-hydroxyestrogen before, during, and after pregnancy in rats. Data points represent the average value $( \pm$ S.E.M.) from four rats. Daily or frequent urine samples were collected from four female rats before, during, and after pregnancy. Samples were tested in duplicate or triplicate in the ESTRAMET ELISA used to measure levels of urinary 2-hydroxyestrogen that were normalized against creatinine. The asterisk indicates that the average value for 2 -hydroxyestrogen on day 16 was statistically significant $(P<0.05)$ when compared with the average values detected in nonpregnant, post partum, and early pregnant rats (up to day 10). The average values of 2-hydroxyestrogen at day 17 and 18 of pregnancy were significantly higher $(P<0.05)$ when compared with average values detected in nonpregnant, post partum, and pregnant rats (up to day 13$)$.

reported for that day of pregnancy (Garfield et al. 1998, Shi et al. 1999). Likewise, values obtained from the pregnant rats treated with the COMT inhibitor matched those typically obtained from rats at day 17 of pregnancy (Garfield et al. 1998, Shi et al. 1999).

\section{Discussion}

Both progesterone and estrogen are considered to be the major factors that regulate uterine function during pregnancy. Progesterone promotes uterine quiescence while estrogen facilitates uterine contractility; however, the mechanisms by which these hormones control uterine function are not completely understood. Results from recent studies suggest that the pathways involved in the metabolism of progesterone or estrogen may also help to regulate the functions of these hormones in the cervix or uterus during pregnancy (Mahendroo et al. 1999, Madsen et al. 2004, Sheehan et al. 2005). Catecholestrogens are the major metabolites produced during estrogen metabolism (Ball \& Knuppen 1980). The catecholestrogens, 2- or 4-hydroxyestrogen, can act as estrogen antagonists (Vandewalle \& Lefebvre 1989, Bradlow 1996, Al-Hendy \& Salama 2006), while their methylated counterparts, 2- or 4-methoxyestrogen, can act as estrogen agonists in multiple biologic assays (Banerjee et al. 2003, Lippert et al. 2003, Liu \& Zhu 2004, Sutherland et al. 2005). In this study, we monitored the longitudinal changes in urinary 2-hydroxyestrogen as an indirect indicator for the changes of estrogen metabolism in pregnant rats before, during, and after pregnancy (Fig. 2). Since COMT activity helps to regulate levels of cellular 2-hydroxyestrogen, we decided to detect and localize expression of the enzyme in uterine tissue during pregnancy as well (Fig. 1).

We found that the levels of rat urinary 2-hydroxyestrogen gradually increased from the beginning of pregnancy, peaked from day 16 to 18 , and then decreased post partum (Fig. 2). The profile generated from our data was similar to the one presented in a previous report, which showed a similar pattern for urinary excretion of 2-hydroxyestrogen during pregnancy in rats (Biswas et al. 1991). A similar pattern for longitudinal changes in urinary 2-hydroxyestrone during human pregnancy has been previously reported (Gelbke et al. 1975). In women, urinary 2-hydroxyestrone reached maximum levels at 26-32 weeks of pregnancy and then decreased during the remainder of the pregnancy (Gelbke et al. 1975). It was also reported that levels of human urinary 2-methoxyestrone were lower in the first trimester of pregnancy and higher near term (Hobkirk \& Nilsen 1963). It should be noted that the values for total human urinary estrogens did not correlate with either the values for 2-hydroxyestrone (Gelbke et al. 1975) or 2-methoxyestrone (Hobkirk \& Nilsen 1963). This indicates that levels for both metabolites did not merely reflect circulating levels of estrogen but, rather, more intricate metabolic steps that regulate the local bioavailability of estrogen.

Results from our immunohistochemistry analyses suggested that COMT expression may be differentially regulated in uterine tissue during pregnancy in the rat (Fig. 1). Levels of COMT expression differed within the $L E$, uterine gland epithelium, endometrial stroma, and myometrium in the days of pregnancy analyzed in the study. High and moderate levels of COMT expression were consistently found within the LE and uterine gland epithelium, while low levels of COMT expression were detected in the EN throughout pregnancy. COMT expression changed within the myometrium during pregnancy as well. Levels of COMT expression were high during the initial days of pregnancy (Fig. 1, day 1 and 2), significantly decreased by midpregnancy (Fig. 1, day 8 and 13), and then increased late in pregnancy (Fig. 1, day 16, 19, and 22). No inverse correlation existed between the levels of urinary 2-hydroxyestrogen 


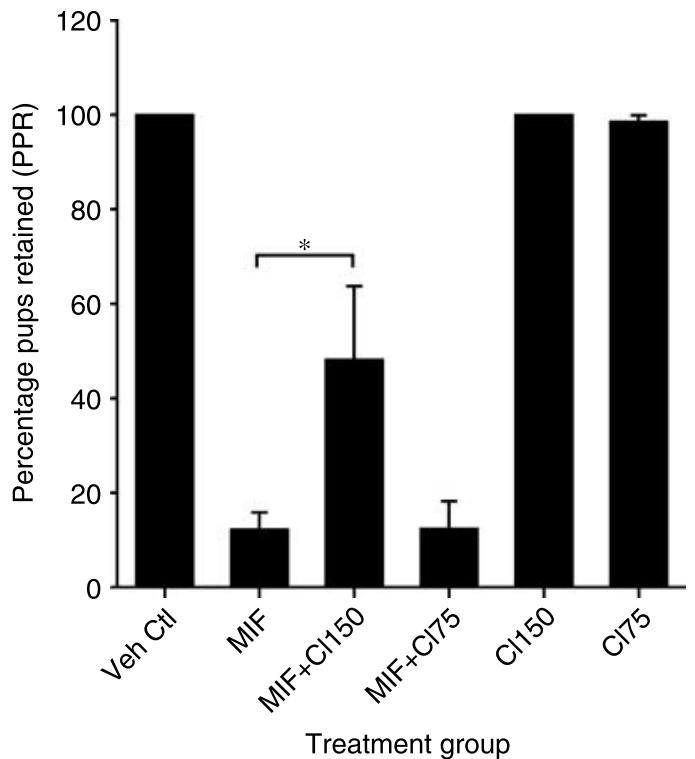

Figure 3 Cotreatment with a selective COMT inhibitor caused an increase in the percentage of pups retained in utero in pregnant rats treated with mifepristone. Bars represent the percentage of pups retained ( \pm S.E.M. value) for each treatment group. Day 17 timedpregnant rats were grouped into six treatment regimens: group 1, vehicle control; group 2, mifepristone alone; group 3, mifepristone+ $\mathrm{Cl150}$; group 4, mifepristone $+\mathrm{Cl} 75$; group 5, Cl150; and group 6, $\mathrm{Cl75}$. Six rats were used for each treatment group. Rats were subcutaneously injected with the selective COMT inhibitor, RO 41 9640 , at a dose of $150 \mathrm{mg} / \mathrm{kg}$ (groups 3 and 5) or $75 \mathrm{mg} / \mathrm{kg}$ (groups 4 and 6). The remaining rats were injected with methylcellulose (groups 1 and 2). Rats were subsequently injected subcutaneously with either mifepristone (10 mg/kg, groups 2-4) or sesame oil (groups 1, 5, and 6). Rats were treated with the COMT inhibitor or methylcellulose every $12 \mathrm{~h}$. All rats in the study were killed at $30 \mathrm{~h}$ post-treatment with the mifepristone and dissected to determine the percentage of pups retained in the uterus, as described in Materials and Methods. The asterisk indicates that the difference in values for the percentage of pups between the mifepristone alone and mifepristone $+\mathrm{Cl} 150$ treatment groups was statistically significant $(P<0.05)$. Veh $\mathrm{Ctl}$, vehicle control; MIF, mifepristone; $\mathrm{Cl}$, COMT inhibitor; 150, $150 \mathrm{mg} / \mathrm{kg}$ dose; 75, $75 \mathrm{mg} / \mathrm{kg}$ dose.

(a COMT substrate; Fig. 2) and the staining intensity scores for COMT protein in the myometrium during pregnancy (Fig. 1). This was not completely unexpected, since the urinary levels of 2-hydroxyestrogen are the net result of global estrogen metabolism from various organs including the liver, kidneys, and central nervous system (Gelbke et al. 1977, Ball \& Knuppen 1980, Tsuchiya et al. 2005). Nevertheless, the general pattern of 2-hydroxyestrogen urinary excretion during pregnancy, which is similar for both rats and humans, suggests that the production and metabolism of 2-hydroxyestrogens may be regulated during pregnancy.

Results from our experiments involving the mifepristone rat model for preterm labor suggested that COMT activity may help to facilitate labor and delivery (Fig. 3). Timed-pregnant rats treated with both mifepristone and $150 \mathrm{mg} / \mathrm{kg}$ of the COMT inhibitor RO 41-0960 had

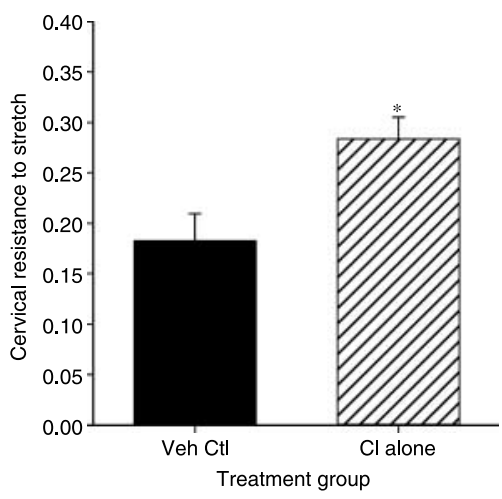

Figure 4 Treatment with a selective COMT inhibitor impeded cervical ripening in pregnant rats. Bars represent average values ( \pm S.E.M.) from three independent experiments. We used three to four rats for each treatment group in each experiment. Day 17 pregnant rats were treated orally with either methylcellulose (vehicle control) or the selective COMT inhibitor, RO 41-0960 (33 mg/kg), every $12 \mathrm{~h}$ for two consecutive days. Rats were euthanized at day 19 of pregnancy and dissected to remove the cervical tissues. A specially designed cervimeter measured the resistance to stretch in the cervices as described in Materials and Methods. An asterisk indicates that the difference in values between the two treatment groups was statistically significant $(P<0.05)$. Veh $\mathrm{Ctl}$, vehicle control; $\mathrm{Cl}$, COMT inhibitor.

a significantly higher PPR in the uterus $(48.2 \pm 15.5$; Fig. 3), when compared with the PPR values calculated for pregnant rats treated with mifepristone alone $(12.2 \pm 3.6$; Fig. 3). Mifepristone is known to induce preterm delivery through an antiprogesterone effect that abolishes the relaxing influence of progesterone in the myometrium. We speculate that the additional treatment with the COMT inhibitor was able to partially block this effect and resulted in a significant increase in the number of pups retained in the uterus (Fig. 3). A high dose of the COMT inhibitor was required to immediately overcome this pronounced effect on uterine contractility because cotreatment with the COMT inhibitor at the $75 \mathrm{mg} / \mathrm{kg}$ dose did not result in an increase in the PPR value (12.4 $\pm 5.8 ;$ Fig. 3). We do not know the mechanism that led to higher retention of pups in the uterus after treatment with the COMT inhibitor. An increase in catechol estrogens and/or catecholamines would reflect inhibition of COMT activity from treatment. We did not measure any of these COMT substrates in our current study; although, data from a previous PTB study showed that treatment with the COMT inhibitor resulted in an increase in the average level of urinary 2-hydroxestrogen (see Supplementary Figure 1, which can be viewed online at http://www.reproduction-online.org/supplemental/). We speculate that treatment blocked COMT enzymatic activity and led to an accumulation of catechol estrogens and possibly catecholamines as well, which influenced the contractile properties of the myometrium.

Levels of circulating progesterone and estrogen did not vary among rats treated with mifepristone alone or 
mifepristone with the inhibitor at the high $(150 \mathrm{mg} / \mathrm{kg})$ or low $(75 \mathrm{mg} / \mathrm{kg})$ doses; therefore, treatment with the COMT inhibitor may have influenced the metabolism and local bioavailability of estrogen. To determine if treatment with the COMT inhibitor caused an alteration in tissue levels of 2-hydroxyestrogen, we plan to conduct an extraction procedure with the myometrium samples that were saved from the pregnant rats used in our study. The extracts will be analyzed by a liquid chromatography/ electrospray ionization tandem mass spectrometry procedure to identify and measure levels of estrogen metabolites. We have successfully used this analytical procedure to evaluate 15 estrogen metabolites, including 2-hydroxyestrogen and 2-methoxyestrogen, in patient samples (Salih et al. 2007b). We propose that COMT activity may help to mediate estrogen-related functions in the myometrium during pregnancy at term. These functions include increases in the levels of factors associated with contractility, such as CX 43 (Mackenzie \& Garfield 1986, Verhoeff et al. 1986, Oltra et al. 2003), oxytocin (Fang et al. 1996), and the receptors for oxytocin (Fang et al. 1996) or PGs (Dong \& Yallampalli 2000). Therefore, treatment with the COMT inhibitor may cause a reduction in one or more of these factors, which in turn could result in the attenuation of uterine contractility induced by mifepristone.

Alternatively, inhibition of COMT activity could alter the metabolism of the catecholamines. Late in pregnancy catecholamines are not locally produced in the uterus due to adrenergic denervation (Thorbert et al. 1979), although circulating levels could act on the $\beta$ and $\alpha$ adrenergic receptors in a non-synaptic manner (Gaspar et al. 1998). In the future, blood samples obtained from the rats in our study will be evaluated to determine if treatment with the COMT inhibitor resulted in an increase in the levels of circulating catecholamines or altered the ratio of epinephrine to norepinephrine, which could affect uterine contractility (Segal et al. 1998).

COMT activity may influence cervical ripening as well (Fig. 4). Treatment of day 17 pregnant rats with the COMT inhibitor for two consecutive days appeared to impede cervical ripening; the average value for resistance to stretch in cervical tissue for these rats was 0.27 (Fig. 4), which is close to values previously reported for day 17 pregnant rats (Garfield et al. 1998, Shi et al. 1999). The average value for the control rats was 0.183 (Fig. 4) and is consistent with values previously reported for day 19 pregnant rats (Garfield et al. 1998, Shi et al. 1999). Data from numerous studies indicate that the action of estrogen contributes to the increase in cell content that is associated with cervical ripening (Ramos et al. 2002, Lee \& Sherwood 2005). It was shown that estrogen, in conjunction with relaxin, promoted cellular proliferation and inhibited apoptosis in cervical epithelium and subepithelium stroma; this effect was more apparent from day 16 to 19 of pregnancy (Lee \& Sherwood 2005). A higher relative distribution of estrogen receptor $\alpha$ was detected within the cervical epithelium and subepithelium stroma during midpregnancy when peaks of cellular proliferation occurred (Ramos et al. 2002). Unlike the rats treated with vehicle control, rats treated with the COMT inhibitor did not appear to undergo further cervical ripening. This may be due to a lower rate of cellular proliferation and higher rate of apoptosis, secondary to lower estrogenic environment and subsequent to inhibited COMT activity. We propose that COMT activity in the cervix may be indirectly involved in cervical ripening by regulating the mitogenic properties of estrogen within the cervical epithelium and subepithelium stroma during pregnancy.

Our results suggest that expression levels of COMT may be differentially regulated in the uterus during pregnancy in the rat. Since COMT is the rate-limiting enzyme in the conversion of 2- or 4-hydroxestrogens to 2- or 4-methoxyestrogens, the regulation of COMT expression may help to control the local action of estrogen. In conclusion, we propose that the regulation of COMT expression may represent one pathway in which the metabolism of estrogen influences uterine and cervical function during pregnancy.

\section{Materials and Methods}

\section{Materials}

Reagents used for the animal studies, which included a selective COMT inhibitor (Ro 41-9640), mifepristone (RU 486), methylcellulose, and sesame oil, were purchased from Sigma Chemicals. Chemicals used for the fixation of the uterine tissues, immunohistochemistry analyses of the tissue sections, and the staining procedure to localize COMTexpression were also purchased from Sigma Chemicals. The anti-COMT polyclonal antibody was kindly provided by J Tenhunen (University of Helsinki, Finland). The secondary antibody which was conjugated to horseradish peroxidase was purchased from Santa Cruz Biotechnology (Santa Cruz, CA, USA).

The ESTRAMET urinary estrogen metabolite kit was purchased from Immuna Care Corporation (Blue Bell, PA, USA).

\section{Animals}

Sprague-Dawley rats were purchased from Harlan Laboratories (Indianapolis, IN, USA). To obtain uterine tissues at different days of pregnancy, two adult female rats were placed with a single male rat in a single cage. On the following morning, mating was confirmed by the presence of spermatozoa in the vaginal fluid and was defined as day 1 of pregnancy. Spontaneous term labor usually occurs between days 22 and 23 of pregnancy in the rat. For the PTB and cervical extensibility studies, Sprague-Dawley rats, timed at day 15 of pregnancy, were purchased and allowed to stabilize for 2 days prior to the study. The pregnant rats were housed separately under controlled light conditions (12 h light: $12 \mathrm{~h}$ darkness) and temperature $\left(23-25^{\circ} \mathrm{C}\right)$. Food and water were available ad libitum. The procedures described for the rat studies were 
approved by the Institutional Animal Care and Use Committee at the University of Texas Medical Branch, Galveston, Texas, USA. At the end of each study, animals were euthanized by carbon dioxide inhalation using a method issued by the American Veterinary Medical Association.

\section{Immunohistochemistry}

Immunohistochemistry was conducted to detect and localize COMT protein expression in rat uterine tissues during pregnancy. Uterine tissues were collected from female rats that were euthanized at days 1, 2, 4, 8, 13, 16, 19, and 22 of pregnancy. Tissues were fixed in PBS solution containing 10\% formalin, embedded in paraffin, and then sectioned. Immunohistochemistry was conducted using standard techniques (Ausubel et al. 1987). The concentration for the anti-COMT polyclonal antibody was 1:1200, and 1:10 000 for the anti-guinea pig (the species used to generate the anti-COMT antibody) secondary antibody, which was conjugated to horseradish peroxidase. The tissue sections were incubated in diaminobenzidine to detect COMT protein and counterstained with hematoxylin. Negative controls included omission of the primary antibody or substitution of the COMT antiserum with normal serum from guinea pigs.

A scoring system was used to quantify the level of COMT expression in the rat myometrium during pregnancy. We chose to analyze the myometrium because we were interested in a putative role of COMT activity in uterine contractility. Four people viewed each immunohistochemistry slide (under the same magnification) and assigned a score, which indicated the intensity of staining, for five random regions. Scores ranged from 0 to 5; where 0 indicated the absence of staining, while a score of 5 indicated intense staining. All of the people were blinded to the day of pregnancy of the rat used to obtain the uterine tissue. The scores for each slide were used in statistical analyses to determine significant differences.

\section{Detection of urinary 2-hydroxyestrogen}

To monitor the longitudinal changes in 2-hydroxyestrogen during pregnancy, four female rats were housed separately in metabolic cages to allow daily collection of urine samples; collection began at preconception, continued during the entire pregnancy, and ended at day 6 post partum. The levels of total 2-hydroxyestrogen (i.e., 2-hydroxyestradiol, 2-hydroxyestrone, 2-hydroxyestriol) in the urine samples were measured with the ESTRAMET urinary estrogen metabolite kit (Immuna Care Corp.), using the protocol provided by the manufacturer. Creatinine levels were also measured in each urine sample and used to normalize the 2-hydroxyestrogen levels.

\section{PTB studies}

We conducted animal studies to determine the effect of treatment with the selective COMT inhibitor, Ro 41-0960 (Sigma Chemicals), on PTB using the antiprogesterone mifepristone (RU 486; Sigma Chemicals) pregnant rat model, which has been shown in numerous studies to be a reliable model for PTB. For example, the treatment of timed-pregnant rats with mifepristone-induced uterine contractility (Buhimschi et al. 1998, Doret et al. 2005) that leads to delivery (Garfield et al. 1987, Shi et al. 2003, Doret et al. 2005). In addition, we and others have shown that treatment with mifepristone caused an increase in contraction-associated proteins such as CX 43 (Mackenzie \& Garfield 1986, Garfield et al. 1987, Miyoshi et al. 1996) and the OTR (Fang et al. 1997) in the myometrium. Finally, delivery induced by mifepristone was shown to occur in a more narrow range of time when compared with the time frame for delivery from spontaneous labor at term (Li et al. 2004).

Day 17 timed-pregnant rats were randomly assigned into six treatment groups with six rats per group. Groups represented one of the following treatment regimens: group 1, vehicle control; group 2, mifepristone alone; group 3, mifepristone plus COMT inhibitor (150 mg/kg); group 4, mifepristone plus COMT inhibitor (75 mg/kg); group 5, COMT inhibitor alone (150 mg/kg); and group 6, COMT inhibitor alone $(75 \mathrm{mg} / \mathrm{kg})$. The mifepristone was prepared in sesame oil and the selective COMT inhibitor, Ro 41-9640, was prepared in 1\% methylcellulose. All rats received treatment through s.c. injections. Rats were initially treated with Ro 419640 at a dose of $150 \mathrm{mg} / \mathrm{kg}$ (groups 3 and 5) or $75 \mathrm{mg} / \mathrm{kg}$ (groups 4 and 6). The remaining rats were treated with $1 \%$ methylcellulose (groups 1 and 2). Rats were then subsequently treated with either mifepristone (10 mg/kg, groups 2-4) or sesame oil (groups 1 , 5 , and 6). Rats were treated with the COMT inhibitor or $1 \%$ methylcellulose every $12 \mathrm{~h}$ until the end of the study. Rats in all six treatment groups were euthanized $30 \mathrm{~h}$ after treatment with mifepristone. This time point was used because it was previously shown to be associated with a high delivery rate of pups induced by treatment with mifepristone at the same dose (Garfield et al. 1987). Each rat was dissected so that the uterus could be opened to count the number of pups in utero. After removing the pups from each uterus, the number of implantation sites was counted to determine the total number of pups carried during pregnancy. Over the years, we have found this method to be more accurate than just counting the number of pups expelled in the cage because of potential cannibalism by the mother (Garfield et al. 1987, Shi et al. 2003). We calculated the percentage of pups retained (PPR) in the uterus for rats in each treatment group using the following equation: $\mathrm{PPR}=$ (total number of pups retained in uterus/total number of implantation sites) $\times 100 \%$.

The PPR value was calculated for each treatment group. These values were used to assess the effect of treatment with the COMT inhibitor on PTBs.

\section{Cervical resistance to stretch studies}

We conducted three animal studies to determine the effect of treatment with Ro 41-0960 (Sigma Chemicals) on cervical resistance to stretch during pregnancy. Cervical resistance to stretch serves as a parameter to determine the extent of ripening in laboratory animals (Garfield et al. 1998). In each study, day 17 timed-pregnant rats were treated with either vehicle control or the COMT inhibitor at a dose of $33 \mathrm{mg} / \mathrm{kg}$, given orally every $12 \mathrm{~h}$ for 2 days. We used three to four rats in each treatment group. A lower dose of the inhibitor was chosen for this experiment because no concomitant treatment with 
mifepristone was used to induce preterm labor. At day 19, all rats were euthanized and the cervices were dissected from each animal for measuring resistance to stretch using a cervimeter as described previously (Chwalisz et al. 1997, Garfield et al. 1998, Shi et al. 1999). The isolated cervices were suspended in organ chambers containing a physiological solution at $37{ }^{\circ} \mathrm{C}$ and bubbled with $95 \% \mathrm{O}_{2}$ and $5 \% \mathrm{CO}_{2}$. The bottom part of the cervix was fixed and the upper part was connected to a force transducer. The cervix was stretched in $0.2 \mathrm{~mm}$ increments at 1-minute intervals, and the resulting force (maximum values) was recorded. During the accommodation periods the cervix reverted back to a relaxed stage, thereby diminishing its resistance to stretch. The tension generated by the cervix upon stretch was recorded by a computer that was connected to the cervimeter. The results were analyzed by CER and CRA software (Schering, Berlin, Germany) as described previously (Chwalisz et al. 1997, Garfield et al. 1998, Shi et al. 1999). The slope of the regression line through the linear portion of the force curve was taken as a parameter of cervical resistance to stretch. Reduction in the slope of the regression line indicates a decrease of cervical resistance to stretch or an increase of its extensibility (Chwalisz et al. 1997, Garfield et al. 1998, Shi et al. 1999).

\section{Statistical analyses}

Data are presented as mean + S.E.M. Scores for the intensity of staining for the COMT protein in the rat myometrium were subjected to Kruskal-Wallis test, followed by the Dunn test (Sigma Stat version 3; SPSS Inc., Chicago, IL, USA). The levels of urinary 2-hydroxyestrogen collected from the nonpregnant, pregnant, and post partum rats were subjected to one-way repeated measures ANOVA followed by the Holm-Sidak multiple comparison procedure (Sigma Stat version 3; SPSS Inc). Data from the mifepristone pregnant rat PTB study were subjected to the $\chi^{2}$ test, followed by pairwise multiple comparisons using contrast statement in Poisson regression analysis (SAS software, Gary, NC, USA). Values of cervical resistance to stretch for pregnant rats treated with the COMT inhibitor versus vehicle control rats were subjected to the Student's $t$-test (Sigma Stat version 3; SPSS Inc). A value of $P<0.05$ was considered statistically significant.

\section{Acknowledgements}

The authors declare that there is no conflict of interest that would prejudice the impartiality of this scientific work.

\section{References}

Al-Hendy A \& Salama SA 2006 Catechol-O-methyltransferase polymorphism is associated with increased uterine leiomyoma risk in different ethnic groups. Journal of the Society for Gynecologic Investigation 13 136-144.

Ausubel FM, Brent R \& Kingston RE 1987 Current Protocols in Molecular Biology, New York: Greene Publishing Associates and WileyInterscience.

Axelrod J \& Tomchick R 1958 Enzymatic O-methylation of epinephrine and other catechols. Journal of Biological Chemistry 233 702-705.
Ball P \& Knuppen R 1980 Catecholoestrogens (2-and 4-hydroxyoestrogens): chemistry, biogenesis, metabolism, occurrence and physiological significance. Acta Endocrinologica 232 1-127.

Banerjee SN, Sengupta K, Banerjee S, Saxena NK \& Banerjee SK 2003 2-Methoxyestradiol exhibits a biphasic effect on VEGF-A in tumor cells and upregulation is mediated through ER- $\alpha$ : a possible signaling pathway associated with the impact of 2-ME2 on proliferative cells. Neoplasia 5 417-426.

Barnea ER, MacLusky NJ, DeCherney AH \& Naftolin F 1988 Catechol-Omethyl transferase activity in the human term placenta. American Journal of Perinatology 5 121-127.

Biswas A, Dale SL, Gajewski A, Nuzzo P \& Chattoraj SC 1991 Temporal relationships among the excretory patterns of 2-hydroxyestrone, estrone, estradiol, and progesterone during pregnancy in the rat. Steroids $\mathbf{5 6}$ 136-141.

Bradlow LH 1996 2-Hydroxyesterone: the 'good' estrogen. Journal of Endocrinology 150 S259-S265.

Briggs MH \& Briggs M 1973 Hormonal influences on erythrocyte catechol-O-methyl transferase activity in humans. Experientia 29 278-280.

Buhimschi I, Yallampalli C, Dong YL \& Garfield RE 1995 Involvement of a nitric oxide-cyclic guanosine monophosphate pathway in control of human uterine contractility during pregnancy. American Journal of Obstetrics and Gynecology 172 1577-1584.

Buhimschi C, Boyle MB, Saade GR \& Garfield RE 1998 Uterine activity during pregnancy and labor assessed by simultaneous recordings from the myometrium and abdominal surface in the rat. American Journal of Obstetrics and Gynecology 178 811-822.

Casey ML \& MacDonald PC 1983 Characterization of catechol-Omethyltransferase activity in human uterine decidua vera tissue. American Journal of Obstetrics and Gynecology 145 453-457.

Casey ML \& MacDonald PC 1988 Biomolecular processes in the initiation of parturition: decidual activation. Clinical Obstetrics and Gynecology 31 533-552.

Casimiri V, Cohen WR, Parvez S, Hobel C \& Parvez H 2004 Phenylethanolamine- $\mathrm{N}$-methyl transferase and catechol-O-methyl transferase activity in rat uterus: cyclic and steroid-induced changes. Acta Obstetricia et Gynecologica Scandinavica 72 606-610.

Castren O \& Saarikoski S 1974 The simultaneous function of catechol-Omethyltransferase and monoamine oxidase in human placenta. Acta Obstetricia et Gynecologica Scandinavica 53 41-47.

Chakraborty C, Davis DL \& Dey SK 1990 The O-methylation of catechol oestrogens by pig conceptuses and endometrium during the periimplantation period. Journal of Endocrinology 127 77-84.

Challis JRG 2000 Mechanism of parturition and preterm labor. Obstetrical and Gynecological Survey $\mathbf{5 5}$ 650-660.

Chwalisz K, Shao-Qing S, Garfield RE \& Beier HM 1997 Cervical ripening in guinea-pigs after a local application of nitric oxide. Human Reproduction 12 2093-2101.

Creveling CR 2003 The role of catechol-O-methyltransferase in the inactivation of catecholestrogen. Cellular and Molecular Neurobiology 23 289-291.

Csapo AI \& Pinto-Dantas CA 1965 The effect of progesterone on the human uterus. PNAS 54 1069-1076.

Dong YL \& Yallampalli C 2000 Pregnancy and exogenous steroid treatments modulate the expression of relaxant $\mathrm{EP}(2)$ and contractile FP receptors in the rat uterus. Biology of Reproduction 62 533-539.

Doret $M$, Bukowski R, Longo $M$, Maul H, Maner WL, Garfield RE \& Saade GR 2005 Uterine electromyography characteristics for early diagnosis of mifepristone-induced preterm labor. Obstetrics and Gynecology 105 822-830.

Fang X, Wong S \& Mitchell BF 1996 Relationships among sex steroids, oxytocin, and their receptors in the rat uterus during late gestation and at parturition. Endocrinology 137 3213-3219.

Fang X, Wong S \& Mitchell BF 1997 Effects of RU486 on estrogen, progesterone, oxytocin, and their receptors in the rat uterus during late gestation. Endocrinology 138 2763-2768.

Farina M, Ribeiro ML, Weissmann C, Estevez A, Billi S, Vercelli C \& Franchi A 2004 Biosynthesis and catabolism of prostaglandin F $2 \alpha$ (PGF2 $\alpha$ ) are controlled by progesterone in the rat uterus during pregnancy. Journal of Steroid Biochemistry and Molecular Biology 91 211-218. 
Ford SP, Christenson LK, Rosazza JP \& Short RE 1992 Effects of Ponderosa pine needle ingestion of uterine vascular function in late-gestation beef cows. Journal of Animal Science 70 1609-1614.

Fuchs AR, Periyasamy S, Alexandrova M \& Soloff MS 1983 Correlation between oxytocin receptor concentration and responsiveness to oxytocin in pregnant rat myometrium: effects of ovarian steroids. Endocrinology $113742-749$.

Garfield RE, Gasc JM \& Baulieu EE 1987 Effects of the antiprogesterone RU 486 on preterm birth in the rat. American Journal of Obstetrics and Gynecology 157 1281-1285.

Garfield RE, Blennerhassett MG \& Miller SM 1988 Control of myometrial contractility: role and regulation of gap junctions. Oxford Reviews of Reproductive Biology 10 436-490.

Garfield RE, Saade G, Buhimschi C, Buhimschi I, Shi L, Shi SQ \& Chwalisz K 1998 Control and assessment of the uterus and cervix during pregnancy and labour. Human Reproduction Update 4 673-695.

Gaspar R, Marki A, Zupko I \& Falkay G 1998 Evidence of non-synaptic regulation of post-partum uterine contractility in the rat. Life Sciences $\mathbf{6 2}$ $1119-1124$.

Geisert RD, Zavy MT, Moffatt RJ, Blair RM \& Yellin T 1990 Embryonic steroids and the establishment of pregnancy in pigs. Journal of Reproduction and Fertility 40 293-305.

Gelbke HP, Bottger M \& Knuppen R 1975 Excretion of 2-hydroxyestrone in urine throughout human pregnancies. Journal of Clinical Endocrinology and Metabolism 41 744-750.

Gelbke HP, Ball P \& Knuppen R 1977 2-Hydroxyoestrogens. Chemistry, biogenesis, metabolism and physiological significance. Advances in Steroid Biochemistry and Pharmacology 6 81-154.

Hobkirk R \& Nilsen M 1963 2-Methoxyestrone as an estrogen metabolite in the human subject. Journal of Clinical Endocrinology and Metabolism 23 274-278.

Hoversland RC, Dey SK \& Johnson DC 1982 Catechol estradiol induced implantation in the mouse. Life Sciences 30 1801-1804.

Kantor BS, Dey SK \& Johnson DC 1985 Catechol oestrogen induced initiation of implantation in the delayed implanting rat. Acta Endocrinologica 109 418-422.

Larcher A, Neculcea J, Breton C, Arslan A, Rozen F, Russo C \& Zingg HH 1995 Oxytocin receptor gene expression in the rat uterus during pregnancy and the estrous cycle and in response to gonadal steroid treatment. Endocrinology 136 5350-5356.

Lee HY \& Sherwood OD 2005 The effects of blocking the actions of estrogen and progesterone on the rates of proliferation and apoptosis of cervical epithelial and stromal cells during the second half of pregnancy in rats. Biology of Reproduction 73 790-797.

Li Y, Je HD, Malek S \& Morgan KG 2004 Role of ERK1/2 in uterine contractility and preterm labor in rats. American Journal of Physiology. Regulatory, Integrative and Comparative Physiology 287 R328-R335.

Liggins GC 1994 Mechanisms of the onset of labour: the New Zealand perspective. Australian and New Zealand Journal of Obstetrics and Gynaecology 34 338-342.

Lippert C, Seeger H \& Mueck AO 2003 The effect of endogenous estradiol metabolites on the proliferation of human breast cancer cells. Life Sciences 72 877-883.

Liu Z-J \& Zhu BT 2004 Concentration-dependent mitogenic and antiproliferative actions of 2-methoxyestradiol in estrogen receptorpositive human breast cancer cells. Journal of Steroid Biochemistry and Molecular Biology 88 265-275.

Mackenzie LW \& Garfield RE 1986 Effects of $17 \beta$-estradiol on myometrial gap junctions and pregnancy in the rat. Canadian Journal of Physiology and Pharmacology 64 462-466.

Madsen G, Zakar T, Manuelpillai U, Wallace E, Kwek K, Yeo GS, Smith R \& Mesiano S 2004 Intracrine control of estrogen action in human gestational tissues at parturition. Journal of the Society for Gynecologic Investigation 11 213-219.

Mahendroo MS, Porter A, Russell DW \& Word RA 1999 The parturition defect in steroid $5 \alpha$-reductase type 1 knockout mice is due to impaired cervical ripening. Molecular Endocrinology 13 981-992.

Miyoshi H, Boyle MB, MacKay LB \& Garfield RE 1996 Voltage-clamp studies of gap junctions between uterine muscle cells during term and preterm labor. Biophysical Journal 71 1324-1334.
Nathanielsz PW 1995 Regulation of the switch from myometrial contractures to contractions in late pregnancy: studies in the pregnant sheep and monkey. Reproduction, Fertility, and Development 7 595-602.

Oltra E, Pfeifer I \& Werner R 2003 Ini, a small nuclear protein that enhances the response of the connexin43 gene to estrogen. Endocrinology 144 3148-3158.

Pakrasi PL \& Dey SK 1983 Catechol estrogens stimulate synthesis of prostaglandins in the preimplantation rabbit blastocyst and endometrium. Biology of Reproduction 29 347-354.

Ramos JG, Varayoud J, Bosquiazzo VL, Luque EH \& Munoz-de-Toro M 2002 Cellular turnover in the rat uterine cervix and its relationship to estrogen and progesterone receptor dynamics. Biology of Reproduction 67 735-742.

Salih SM, Salama SA, Fadl AA, Nagamani M \& Al-Hendy A 2007 a Expression and cyclic variations of catechol-O-methyl transferase in human endometrial stroma. Fertility and Sterility [in press].

Salih S, Xu X, Veenstra TD, Duleba AJ, Fouad H, Nagamani M \& AlHendy A $2007 b$ Lower levels of urinary 2-hydroxyestrogens in polycystic ovary syndrome. Journal of Clinical Endocrinology and Metabolism 92 3285-3291.

Segal S, Csavoy AN \& Datta S 1998 The tocolytic effect of catecholamines in the gravid rat uterus. Anesthesia and Analgesia 87 864-869.

Sheehan PM, Rice GE, Moses EK \& Brennecke SP 20055 -dihydroprogesterone and steroid $5 \beta$-reductase decrease in association with human parturition at term. Molecular Human Reproduction 11 495-501.

Shi L, Shi SQ, Saade GR, Chwalisz K \& Garfield RE 1999 Changes in cervical resistance and collagen fluorescence during gestation in rats. Journal of Perinatal Medicine 27 188-194.

Shi L, Shi SQ, Given RL, von Hertzen H \& Garfield RE 2003 Synergistic effects of antiprogestins and iNOS or aromatase inhibitors on establishment and maintenance of pregnancy. Steroids 68 1077-1084.

Spicer LJ \& Hammond JM 1988 Effects of 2-hydroxyestradiol on the number of granulosa cell $\beta$-adrenergic receptors. Endocrinology 122 2710-2717.

Spicer LJ \& Hammond JM 1989 Regulation of ovarian function by catecholestrogens: current concepts. Journal of Steroid Biochemistry 33 489-501.

Sutherland TE, Schuliga M, Harris T, Eckhardt BL, Anderson RL, Quan L \& Stewart AG 2005 2-methoxyestradiol is an estrogen receptor agonist that supports tumor growth in murine xenograft models of breast cancer. Clinical Cancer Research 11 1722-1732.

Tekpetey FR \& Armstrong DT 1994 Catecholestrogen modulation of steroid production by rat luteal cells: mechanism of action. Molecular and Cellular Endocrinology 101 49-57.

Tezuka N, Ali M, Chwalisz K \& Garfield RE 1995 Changes in transcripts encoding calcium channel subunits of rat myometrium during pregnancy. American Journal of Physiology. Cell Physiology 269 C1008-C1017.

Thorbert G, Alm P, Bjorklund AB, Owman C \& Sjoberg NO 1979 Adrenergic innervation of the human uterus. Disappearance of the transmitter and transmitter-forming enzymes during pregnancy. American Journal of Obstetrics and Gynecology 135 223-226.

Tsuchiya Y, Nakajima M \& Yokoi T 2005 Cytochrome P450-mediated metabolism of estrogens and its regulation in human. Cancer Letters 227 $115-124$.

Vandewalle B \& Lefebvre J 1989 Opposite effects of estrogen and catecholestrogen on hormone-sensitive breast cancer cell growth and differentiation. Molecular and Cellular Endocrinology 61 239-246.

Verhoeff A, Garfield RE, Ramond J \& Wallenburg HC 1986 Electrical and mechanical uterine activity and gap junctions in estrogen-treated oophorectomized sheep. American Journal of Obstetrics and Gynecology 155 1192-1196.

Wentz MJ, Jamaluddin M, Garfield RE \& Al Hendy A 2006 Regulation of catechol-O-methyltransferase expression in human myometrial cells. Obstetrics and Gynecology 108 1439-1447.

Received 25 May 2007

First decision 19 June 2007

Revised manuscript received 30 July 2007

Accepted 3 September 2007 